\title{
Podoplanin and PROX1 Expression in Hypercaloric Diet-induced Pancreatic Injuries
}

\author{
DELIA TARAN ${ }^{1}$, VALERIA NICOLETA TARLUI ${ }^{1,2}$, RALUCA AMALIA CEAUSU $^{1,2}$, \\ ANCA MARIA CIMPEAN ${ }^{1,2}$, MARIUS RAICA ${ }^{1,2}$ and SIMONA SARB ${ }^{1,2}$ \\ ${ }^{1}$ Department of Microscopic Morphology/Histology, \\ Victor Babes University of Medicine and Pharmacy, Timisoara, Romania; \\ ${ }^{2}$ Angiogenesis Research Center, Victor Babes University of Medicine and Pharmacy, Timisoara, Romania
}

\begin{abstract}
Background: The role of podoplanin (PDPN) and homebox prospero gene 1 (PROXI) in early stages of pancreatic islet changes induced by hypercaloric diet is unclear. The aim of this study was to study PDPN and PROX1 variability in pancreatic islets after a hypercaloric diet in a rat experimental model. Materials and Methods: Pancreatic biopsies harvested from Sprague-Dawley rats at 3, 6, and 9 weeks following hypercaloric diet intake were evaluated for morphological and molecular changes of Langerhans islets based on PDPN and PROXI expression Results: Six weeks of hypercaloric diet induced hypertrophy of pancreatic islets with focal expression of Pdpn and Proxl mRNA. At 9 weeks of hypercaloric diet, strong periinsular inflammation was found around hypertrophic islets highly expressing PDPN, and lacking Proxl $m R N A$ and protein expression. Conclusion: This is the first report of Pdpn and Proxl mRNA expression variability and involvement in early steps of pancreatic islet changes following hypercaloric food intake.
\end{abstract}

Obesity represents the leading cause of morbidity and mortality. The risk of developing obesity-related complications such as cardiovascular disease, stroke, type 2 diabetes and various types of cancer is increased in the obese population. More than $36.5 \%$ of US adults suffer from this chronic disease (1).

This article is freely accessible online.

Correspondence to: Professor Anca Maria Cimpean, MD, Ph.D., Department of Microscopic Morphology/Histology, Angiogenesis Research Center, Victor Babes University of Medicine and Pharmacy, Piata Eftimie Murgu no. 2, 300041, Timisoara, Timis, Romania. Tel: +40 256204476, e-mail: ancacimpean1972@yahoo.com

Key Words: Podoplanin, PROX1, pancreatic island, hypercaloric diet.
Literature data show that obesity may favor lymphedema development and enhancement of inflammatory responses (2-5).

Wang et al. demonstrated that homebox prospero gene 1 (PROX1) is necessary for endocrine progenitor formation and alpha cell differentiation and is expressed at high levels in all pancreatic endocrine progenitors (6). PROX1 is expressed in the adult pancreas at variable levels, with high values in the cells located in the islet mantle: $\alpha$-cells, $\delta$-cells, pancreatic polypeptide secreting cells, and $\varepsilon$-cells. It was found that PROX1 expression was required for $\beta$-cell differentiation (7). Paul et al., in a murine experimental model, demonstrated that high levels of PROX1 drastically impaired $\beta$-cell maturation and expansion (8).

Well-known as a marker for lymphatic endothelial cells, podoplanin has been shown to be a diagnostic marker for many tumor types, such as seminoma, melanoma and squamous-cell carcinoma (9). Podoplanin-positive lymphatic vessels were reported in the pancreas of a single human fetus of 18 gestational weeks. The time of lymphatic colonization and interrelation with blood vessel formation in the pancreas is unknown (10). In normal tissues, podoplanin is a marker of basal cells in the layered epithelium of the skin and cervix, or is intensively expressed in several simple epithelia, such as the alveolar epithelium and the renal tubules (9). Podoplanin involvement in pancreatic development is less studied, and even lesser in diabetes-induced tissue damage. Few data have been reported regarding podoplanin expression in neuroendocrine carcinomas of pancreatic origin (9).

Consequently, the aim of this work was to study podoplanin and PROX1 expression in normal and modified pancreatic tissue, after hypercaloric diet, in a murine experimental model.

\section{Materials and Methods}

Animal model. The present study included 12 4-week-old Sprague Dawley rats assigned to two distinct subgroups, six rats each, with one diet and 3 three time points. The first subgroup was used to see 
in vivo $33: 1157-1163(2019)$

Table I. Hypercaloric diet components, amounts, caloric values and schedule of administration for the groups receiving high fat and carbohydrate foods used to induce the cellular changes of the pancreas as possible substrates for diabetes.

\begin{tabular}{lccc}
\hline Hypercaloric diet components & Food amount, $\mathrm{g}$ & Caloric value/100 g, kcal & Food intake schedule \\
\hline Hamburger & 200 & 498 & 3 Times per week \\
French fries & 100 & 400 & 3 Times per week \\
Ham & 50 & 294 & Daily \\
High fat maturated cheese & 50 & 300 & Daily \\
Salami & 50 & 245 & Daily \\
Sausages & 50 & 450 & Daily \\
Chocolate & 30 & 500 & Daily \\
Sweets & 100 & $600-800$ & Daily \\
\hline
\end{tabular}

if hypercaloric diet induces structural changes inside pancreatic tissue and the second one to replicate the experiment in order to validate the results obtained from the first group. One further animal was used as control and received normo-caloric food for rats. Other rats received foods similar to those consumed by humans: high-fat cheese, salami, sausages, bacon together with sweets containing highly refined sugar. In addition, three times per week, the rats received a fast-food menu containing French fries and high-fat hamburgers. All food was chosen to be similar to food consumed by humans knowing that such food is incriminated as one of the main causes of type 2 diabetes, able to induce inflammation and pancreatic islet damage. Details regarding diet composition and caloric value are given in Table I. We avoided any chemicals known to produce damage to pancreatic islets because we wanted to determine whether there would be morphological changes of the pancreas using food similar to that consumed by humans (type 2 diabetes not being induced by chemicals in normal life).

Pancreatic biopsies were harvested from Sprague-Dawley rats at 3,6 , and 9 weeks of a hypercaloric diet and were evaluated for morphological and molecular changes of Langerhans islets.

Immunohistochemistry. Pancreatic specimens collected from the experimental rats on the hypercaloric diet described, together with normal pancreas harvested from the control rat, were routinely paraffin embedded and stained with hematoxylin and eosin for the preliminary morphological evaluation of these changes. This step was followed by selection of samples for the immunohistochemical staining. Immunohistochemical technique was performed with the Leica Bond-Max Autostainer (Leica Biosystems, Newcastle upon Tyne, UK). Briefly, immunohistochemical procedure was chosen by selection of the proper protocol from autostainer software. This protocol includes all steps of immunohistochemistry from automated dewaxing, epitope retrieval by using high $\mathrm{pH}$ buffer solution Novocastra Bond Epitope Retrieval Solution 2 (Leica Biosystems) for 20 minutes, endogenous peroxidase inhibition with hydrogen peroxide $(3 \%)$ followed by incubation with the primary antibodies for podoplanin (monoclonal mouse, anti-human, clone D2-40; RTU, Dako Flex, incubation for $30 \mathrm{~min}$; Dako, Glostrup, Denmark) and PROX1 (60 min incubation time; RELIATech $\mathrm{GmBH}$, Wolfenbuttel, Germany). Visualization was achieved using 3,3'-diaminobenzidine as chromogen (for $10 \mathrm{~min}$ ), while hematoxylin was applied for $5 \mathrm{~min}$ for counterstaining. Brown staining of the specimens was considered positive and was evaluated according to its intensity and distribution inside the cells (with cytoplasmic/nuclear pattern).

RNAscope in situ hybridization. In situ hybridization was used to identify RNA and DNA on paraffin-embedded sections. The RNAscope method was used to evaluate the RNA cellular content as visualization of a single molecule in individual cells on formalinfixed and paraffin-embedded specimens (FFPE) using a new sample design strategy and a signal amplification system based on hybridization in order to achieve simultaneous signal amplification and field suppression. Double Z samples for Prox 1, complementary to RNA targeting, was performed by Advanced Cell Diagnostics (Hayward, CA, USA). The RNAscope High-Definition kit (Advanced Cell Diagnostics) was used to amplify the signal, followed by visualization of amplified signals with diaminobenzidine as chromogen. Briefly, in order to prepare FFPE specimens for the RNAscope procedure, the tissues were fixed and permeabilized to allow access to the target sample. The next step was hybridization for $2 \mathrm{~h}$ at $40^{\circ} \mathrm{C}$, followed by multistep signal amplification using reagents, and by RNA visualization as brown dots stained with diaminobenzidine. Detection of the sensitivity of the RNAscope method was demonstrated by using positive DNAdirected RNA polymerase II subunit RPB1 (POLR2A) and negative (targeted against $\operatorname{dap} B$ bacterial gene) controls evaluated using the same protocol. The semi-quantitative interpretation of podoplanin (Pdpn) and homebox prospero gene 1 (Proxl) mRNA amplification was manually performed, according to the score guides provided by the manufacturer. Pdpn mRNA and Proxl mRNA amplification was classified into five groups as: 0: No coloration or fewer than one dot for every 10 cells at $40 \times$ magnification; 1 : 1-3 dots/cell visible at $\times 20-40$ magnification; $2: 4-10$ dots/cell, very few dot clusters visible at $\times 20-40$ magnification; $3:>10$ dots/cell, $<10 \%$ positive cells with dot clusters visible at $20 \times$ magnification; and $4:>10$ dots/cell, more than $10 \%$ positive cells with dot clusters visible at $20 \times$ magnification.

Examination and analysis of microscopic images. The microscope slides were scanned using a Pannoramic Desk Scanner (3DHistech, Budapest, Hungary) and were then loaded onto the Case Center database of the Histology Department. In order to visualize all processed slides, the Pannoramic Viewer system was used, which allows the examination of the entire scanned section. Detailed images were taken using the same system and were saved as JPEG files. 

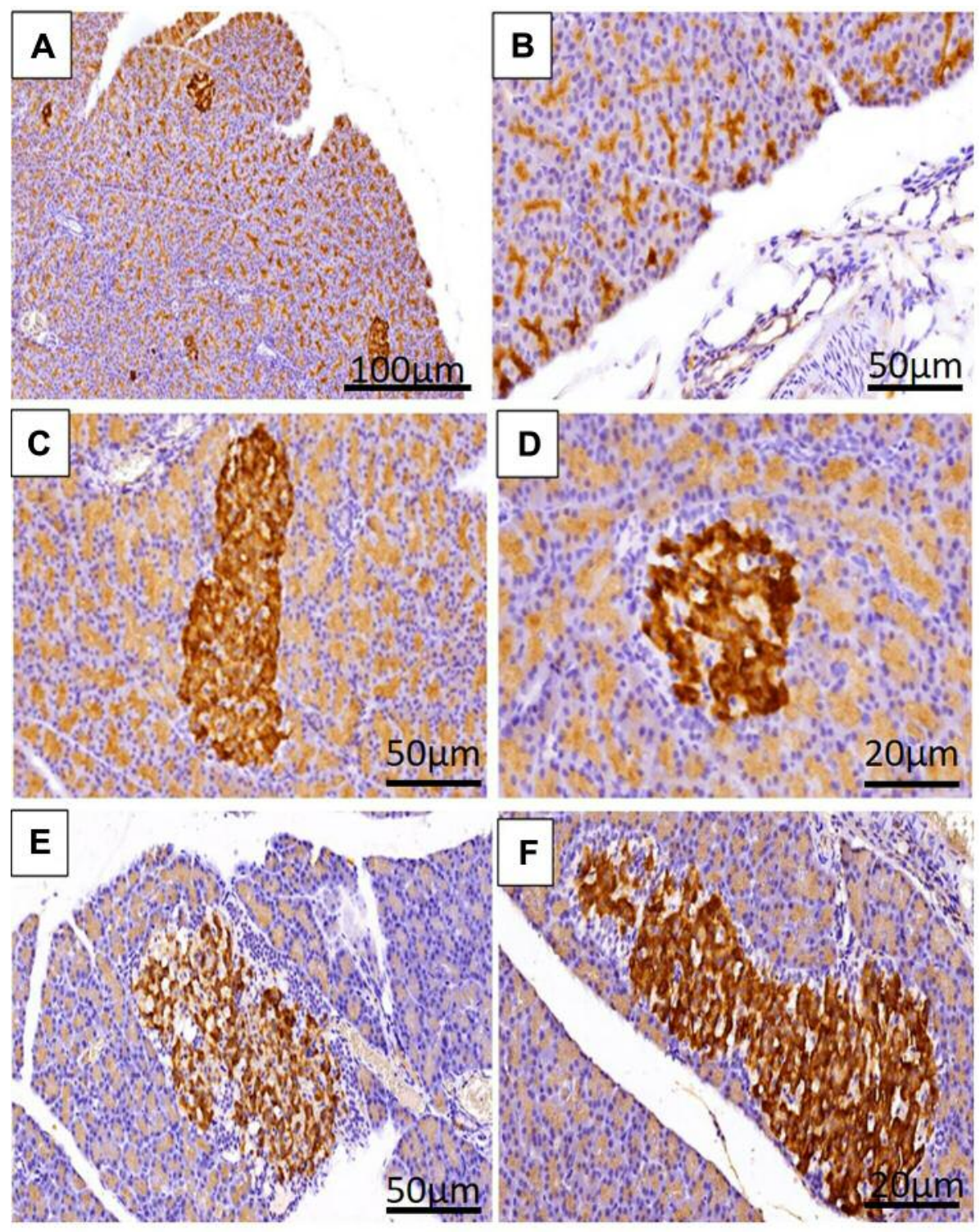

Figure 1. A: Overview of homogeneous podoplanin expression in Langerhans islets and centroacinar ducts, with a more intense expression the peripheral pancreatic lobules, for normal pancreas from the control ( $\times 40$ magnification). B: Image showing the expression of podoplanin in centroacinar cells and endothelial lymphatic cells from peripancreatic adipose tissue for normal pancreas from the control ( $\times 100$ magnification). $C$ and D: Podoplanin expression in the islets became heterogeneous, with increased intensity at the periphery of Langerhans islet for specimens from rats after 6 weeks of a hypercaloric diet ( 400 magnification). E: Increased podoplanin expression in hypertrophic islets, with rich peri-islet inflammatory infiltrate in a rat after 6 weeks of a hypercaloric diet. F: Increased podoplanin expression can be seen in a hypertrophic islet with peri-islet inflammatory infiltrate in a specimen from a rat after 9 weeks of a hypercaloric diet ( $\times 200$ magnification).

\section{Results}

In normal pancreatic tissue, podoplanin immunoexpression was noted mainly in Langerhans islands. In exocrine pancreas, expression was found in the ducts of the centroacinar zone, with higher intensity of expression in the pancreatic periphery. Inside the Langerhans islands of normal pancreas, podoplanin was expressed with higher intensity and a homogeneous distribution throughout the entire island (Figure 1A).

Lymphatic vessels were not found in pancreatic islands nor in the exocrine pancreatic septa, but were noted in peripancreatic connective tissue (Figure 1B). Podoplanin expression, with the previously noted features, was recorded 


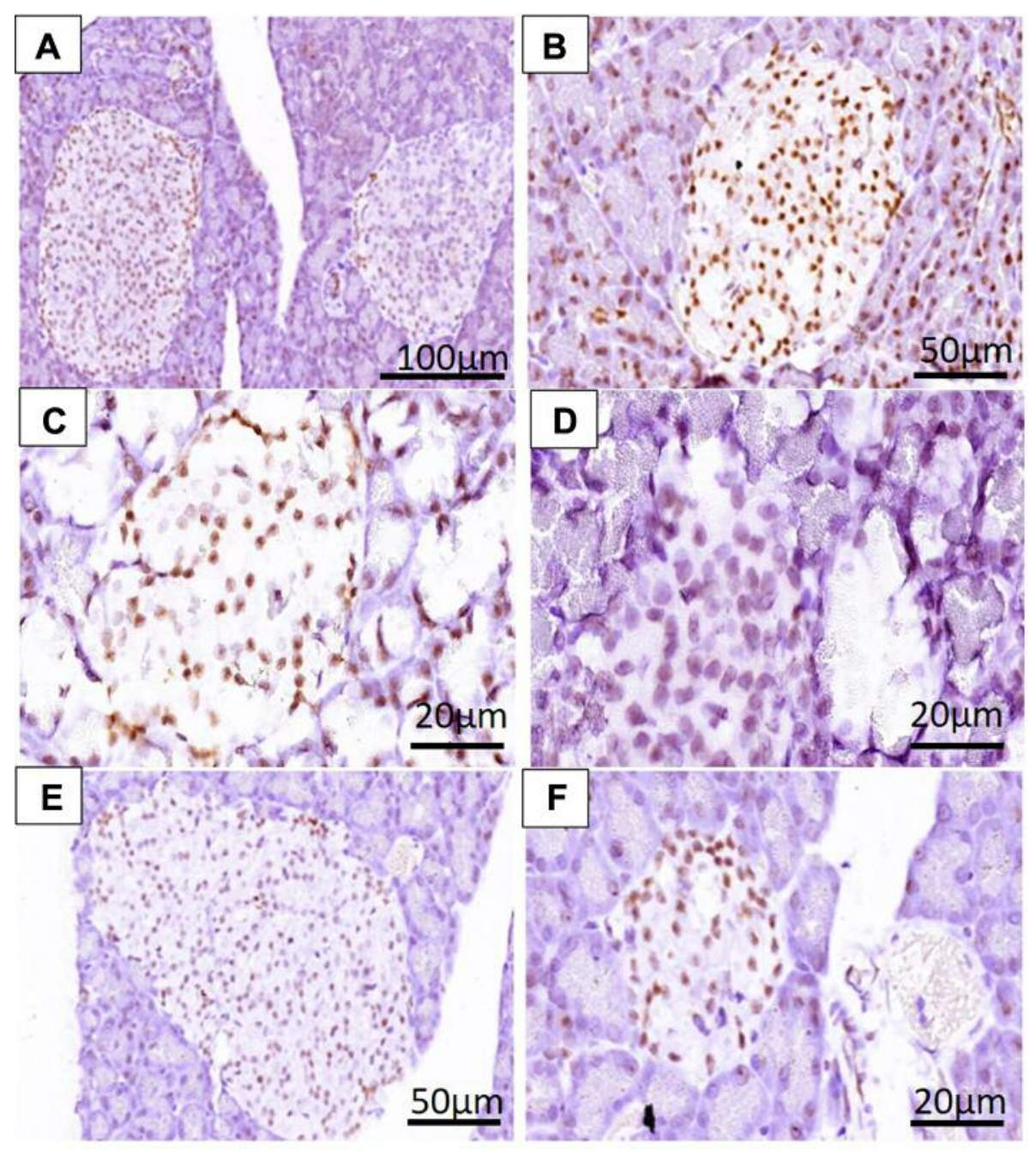

Figure 2. Podoplanin expression visualized by the RNAscope method. A: Positive cells highlighted at the periphery of islets from a rat after 6 weeks of a hypercaloric diet (x40 magnification). B: Podoplanin mRNA expression in islets from a rat after 9 weeks of a hypercaloric diet ( $\times 400$ magnification). C: Cells at the periphery of pancreatic islets were highly positive for homebox prospero gene 1 (Prox1) for specimens of normal pancreas from the control ( $\times 400$ magnification). D: For a specimen from a rat after 3 weeks of a hypercaloric diet, Prox1-positive cells decreased at the islet periphery, but rare positive cells were also observed in central areas ( $\times 400$ magnification). E: In rats after 6 weeks of a hypercaloric diet, the distribution of Proxl-positive islet cells was focal, in small groups at the islet periphery. In the central area of islets, expression was isolated and with high density, both in hypertrophic islets and normal areas, with cluster expression of Prox 1 mRNA in hypertrophic islets $(\times 200$ magnification). F: In normal-sized islets of a specimen after 6 weeks of a hypercaloric diet, the distribution of positive cells was different from that of normal pancreas from the control (density of central positive cells was high) ( $\times 200$ magnification).

in compact and diffuse islands and in isolated endocrine cells from the excretory ducts.

After 3 weeks of hypercaloric nutrition, some changes in podoplanin expression were noted in pancreatic islands. Expression was found to be heterogeneous, with higher intensity in the periphery compared with the central area of Langerhans islands, in 50-70\% of Langerhans island cells (Figure $1 \mathrm{C}$ and D).

After 6 weeks of hypercaloric nutrition, podoplanin expression significantly decreased in both hypertrophic islands and in relatively normal areas. A heterogeneous pattern of density and intensity of expression was found.

Most of the cells in Langerhans islands were negative or weakly positive and only a small proportion of cells, stained moderately or strongly positive.

A higher intensity of podoplanin expression was found in up to $50 \%$ of hypertrophic islet cells, although only in those islets in which a rich inflammatory infiltrate was found (Figure 1E).

Nine weeks after the initiation of hypercaloric diet most islets were hypertrophic, whereas the inflammatory infiltrate 
was consistently seen around them, and increased podoplanin expression in hypertrophic islets with rich inflammatory infiltrate was noted (Figure 1F).

Differentiated expression of podoplanin was verified using the RNAscope method. By this method we were able to see Pdpn mRNA expression in the Langerhans islet cells at 6 and 9 weeks of the hypercaloric diet. The intensity and distribution were consistent with the immunohistochemical expression of podoplanin. RNAscope confirmed that peripheral islet cells from mice 6 weeks of the hypercaloric diet expressed PDPN (Figure $2 \mathrm{~A}$ and $\mathrm{B}$ ).

In the normal pancreas, Proxl mRNA was noted to be intensely expressed in the cells at the periphery of Langerhans islets and rarely in the central islet cells (Figure 2C).

In pancreas of mice after 3 weeks of the hypercaloric diet, Proxl expression was seen to have decreased in peripheral islet cells, while in the other cells (no more than $50 \%$ of the total), expression was maintained, although with a +1 score, with spot amplitude, but not exceeding 1-2 dots/cell (Figure 2D).

In pancreas collected after 6 weeks of the hypercaloric diet, focal distribution of Proxl-positive cells in small groups at the periphery, with cluster expression of Proxl mRNA was seen. Prox 1 mRNA had a +2 score in the remaining islets. In normal-sized islets, the distribution of Proxl-positive cells was similar to that in the normal pancreas (Figure $2 \mathrm{E}$ and $\mathrm{F}$ ). In the pancreas after 9 weeks administration of hypercaloric food, the expression of Proxl was negative, with only isolated peripheral cells (1-2/islet) expressing Prox1.

\section{Discussion}

Podoplanin, lymphatic vessel endothelial hyaluronan receptor 1 (LYVE1) and PROX1 are recognized markers of normal and tumor lymph vessels. These three markers are still extensively used to quantify lymphatic microvessel density in tumors, although they were shown to have only a minimal impact on the evaluation of lymphatic metastases (9). Many literature data have reported and described the expression of lymphatic markers in pancreatic endocrine tumors. In 2007, Tomita published the first description of expression of a lymphatic marker in pancreatic endocrine cells. Tomita described LYVE1 expression in all normal pancreatic islets and its decreased expression in endocrine pancreatic neoplasms (10). In 2009, the same author performed immunostaining with D2-40 for podoplanin on the same normal and tumor pancreatic tissue and reported its expression in normal pancreatic islets (11). Other lymphangiogenic factors, such as vascular endothelial growth factor-C and -D, were identified in pancreatic endocrine cells before Tomita's report, and were described by Kerjaschki's team (12). Podoplanin expression in the pancreas was recently extensively studied in stromal fibroblasts associated with various types of pancreatic tumors (13).
Podoplanin involvement in the pathogenesis of diabetes mellitus has not been reported so far in endocrine and exocrine pancreas. Our results confirmed podoplanin expression in normal pancreatic endocrine cells in normal pancreas and supported expression of podoplanin in exocrine pancreas, restricted to the apex of serous pancreatic cells. As podoplanin expression in endocrine pancreas has barely been studied in the literature, we deemed it appropriate to confirm its expression by RNAscope in situ hybridization, which validated the immunohistochemistry results.

For this reason, we continued the study on pancreas harvested at 3, 6 and 9 weeks, respectively. Marked heterogeneity of podoplanin expression according to the duration of the hypercaloric diet, as well as the presence or absence of inflammatory infiltrate in the pancreatic endocrine islets, was seen, which characterized the late stages of the experiment.

A specific feature was observed at this point in the experiment, the heterogeneity of podoplanin expression, which was dependent on the presence of peri- and intrainsular inflammatory infiltrate. Podoplanin expression in islet endocrine cells most likely triggers the activation of C-type lectin domain family 1 member B (CLEC2) receptor which is sensitive to changes in glucose level, activates the immune and autoimmune mechanisms described in other organs, and many contribute to irreversible destruction of pancreatic islets. This is supported by our observation that podoplanin remained highly expressed at 9 weeks from hypercaloric diet initiation mainly in Langerhans islets which associated a strong inflammatory infiltrate. RNAscope confirmed that podoplanin expression in pancreatic endocrine cells is not aberrant, although further studies are needed to clarify the role of podoplanin in the pathogenesis of diabetes mellitus.

Podoplanin transcription is enhanced by PROX1 in lymphatic endothelial cells (14) in all species as a mandatory step in lymphatic endothelial cells differentiation. PROX1 is normally expressed in the peripheral cells of pancreatic islets, which are glucagon-secretoring cells in adult life, as well as in prenatal pancreatic immature beta cells. Our study demonstrated mRNA expression of Proxl in endocrine pancreatic islets. A decrease of PROX1 expression stimulates the expansion and maturation of pancreatic $\beta$-cells, whilst its overexpression alters glucose homeostasis in immature $\beta$ cells (14). Paul and co-workers demonstrated that Proxl overexpression in immature $\beta$-cells leads to hyperglycemia and hypo-insulinemia (8). The same team also reported Proxl overexpression to be 9- to 13-fold higher in the pancreas of hyperglycemic mice as compared to normal pancreatic $\beta$-cells.

PROX1 overexpression in immature pancreatic $\beta$-cells leads to a decrease in islet $\beta$-cell proliferation, which might partly explain the inability of pancreatic $\beta$-cells to regenerate under a hypercaloric diet, thus contributing to the 
maintenance of pancreatic islet destruction in diabetes (8). The increase and persistence of PROX1 expression in pancreatic islet cells interferes with maturation of pancreatic $\beta$-cells, which may partly explain the lack of pancreatic islet regeneration in diabetes.

The finding of two Proxl-dependent phenotypes, 'normoglycemic' versus 'hyperglycemic', was another interesting observation of the study published by Paul and coworkers (8) who proved that a decrease in PROX1 regulation is a prerequisite for the expansion of $\beta$-cell mass after birth and for proper maturation of this filiation. Our findings substantiate the study of PROX1 expression using current protocols for directed differentiation of insulin secretory cells from humaninduced pluripotent stem cells or embryonic stem cells and suggests that manipulation of levels of this transcription factor might increase the production of responsive beta cells at increased glucose levels, which is useful for therapy. Finally, the results of genome-association studies identifying single nucleotide polymorphism in the $P R O X 1$ regulatory regions correlating with diabetes tendency, suggesting PROXI involvement in pathogenesis and progression of diabetes (8).

Our study shows that PROX1 expression following morphological changes of the pancreas during a hypercaloric diet is extremely heterogeneous, being highest in the $\beta$-cells located in the center of islets at 6 weeks of the hypercaloric diet. This can be explained by the pancreas attempting to regenerate islet $\beta$-cells, and for this reason, PROX1-positive cells in the center of islets can be considered immature $\beta$-cells.

One aspect to be discussed regarding the expression of PROXI mRNA in our study would be that of the lack of expression for specimens taken after 9 weeks of the hypercaloric diet. This might be partly explained by the destruction of pancreatic islets at this stage, most likely by an immune/autoimmune mechanism, as we mentioned. Several studies identified PROX1 as being responsible for glucose homeostasis, with an important involvement in the pathophysiology of diabetes. Lecompte et al. mentioned that an increase in glucose level suppresses expression of PROX1 mRNA but not of its protein (15). The same authors showed that PROX1 is involved in the regulation of insulin secretion. $P R O X 1$ repression led to severe decrease in insulin secretion in that study (15). Our findings are similar with these observations. Once PROXI mRNA expression disappeared, the serum glucose level significantly increased, and immunohistochemical expression of insulin was reduced. All these data support the idea that the mechanism regulating excess insulin secretion was overwhelmed, thus being unable to compensate for the increased insulin requirements due to the hypercaloric diet.

\section{Conclusion}

Our study demonstrated the involvement of the podoplanin/ PROX1 axis in the irreversible changes of endocrine pancreatic parenchyma. Both podoplanin and PROX1 were expressed differently, depending on the duration of the hypercaloric diet. Podoplanin mediates the destruction of pancreatic islets, most probably by an immune/autoimmune mechanism.

\section{Conflicts of Interest}

The Authors declare that they have no conflict of interests in regard to this study

\section{Authors' Contributions}

Delia Taran designed the study and write the article; Anca Maria Cimpean and Marius Raica supervised microscopic, morphological and immunohistochemical procedures, and independently evaluated the specimens; Amalia Raluca Ceausu performed immunohistochemistry; Valeria Nicoleta Tarlui and Simona Sarb took care of animals and revised the article.

\section{Acknowledgements}

The Authors are grateful to Victor Babes University of Medicine and Pharmacy Timisoara, and would like to thank to our histotechnologists Patricia Berzava and Ciprian Onica for their excellent technical support.

\section{References}

1 Segula D: Complications of obesity in adults: A short review of the literature. Malawi Med J 26: 240-244, 2014. PMID: 24959321.

2 Greene AK, Grant FD and Slavin SA: Lower-extremity lymphedema and elevated body-mass index. N Engl J Med 366: 2136-2137, 2012. PMID: 22646649. DOI: 10.1056/NEJMc 1201684

3 Arngrim N, Simonsen L, Holst JJ and Bülow J: Reduced adipose tissue lymphatic drainage of macromolecules in obese subjects: A possible link between obesity and local tissue inflammation. Int J Obes 37: 748-750, 2013. PMID: 22751255. DOI: 10.1038/ ijo. 2012.98

4 Nitti MD, Hespe GE, Kataru RP, García Nores GD, Savetsky IL, Torrisi JS, Gardenier JC, Dannenberg AJ and Mehrara BJ: Obesity-induced lymphatic dysfunction is reversible with weight loss. J Physiol 594: 7073-7087, 2016. PMID: 27619475. DOI: $10.1113 / \mathrm{JP} 273061$

5 Ariyagunarajah R and Chen $\mathrm{HH}$ : To be or not to be obese: Impact of obesity on lymphatic function. J Physiol 595(5): 14491450, 2017. PMID: 28247512. DOI: 10.1113/JP273743

6 Wang J, Kilic G, Aydin M, Burke Z, Oliver G and Sosa-Pineda B: PROX1 activity controls pancreas morphogenesis and participates in the production of "secondary transition" pancreatic endocrine cells. Dev Biol 286(1): 182-194, 2005. PMID: 16122728. DOI: 10.1016/j.ydbio.2005.07.021.

7 Westmoreland JJ, Kilic G, Sartain C, Sirma S, Blain J, Rehg J, Harvey $\mathrm{N}$ and Sosa-Pineda B: Pancreas-specific deletion of PROX1 affects development and disrupts homeostasis of the exocrine pancreas. Gastroenterology 142(4): 999-1009, 2012. PMID: 22178591. DOI: 10.1053/j.gastro.2011.12.007 
8 Paul L, Walker EM, Drosos Y, Cyphert HA, Neale G, Stein R, South J, Grosveld G, Herrera PL and Sosa-Pineda B: Lack of Prox 1 down-regulation disrupts the expansion and maturation of postnatal murine $\beta$-cells. Diabetes 65(3): 687-698, 2015. PMID: 26631740. DOI: $10.2337 / \mathrm{db} 15-0713$

9 Raica M, Cimpean AM and Ribatti D: The role of podoplanin in tumor progression and metastasis. Anticancer Res 28(5B): 29973006, 2008. PMID: 19031946.

10 Tomita T: Lymphatic vessel endothelial hyaluronan receptor 1 immunocytochemical staining for pancreatic islets and pancreatic endocrine tumors. Pancreas 35(4): 18-22, 2007. PMID: 18090227. DOI: 10.1097/MPA.0b013e318068fcba

11 Tomita T: D2-40 immunocytochemical staining for pancreatic islets and pancreatic endocrine tumors. Pancreas 38(3): 339-340, 2009. PMID: 19307929. DOI: 10.1097/MPA.0b013e3181975b8c

12 Sipos B, Klapper W, Kruse M-L, Kalthoff H, Kerjaschki D and Klöppel G: Expression of lymphangiogenic factors and evidence of intratumoral lymphangiogenesis in pancreatic endocrine tumors. Am J Pathol 165(4): 1187-1197, 2004. PMID: 15466 385. DOI: $10.1016 / \mathrm{S} 0002-9440(10) 63379-2$
13 Hirayama K, Kono H, Nakata Y, Akazawa Y, Wakana H, Fukushima $\mathrm{H}$ and Fujii $\mathrm{H}$ : Expression of podoplanin in stromal fibroblasts plays a pivotal role in the prognosis of patients with pancreatic cancer. Surgery Today 48(1): 110-118, 2018. PMID: 28702871. DOI: 10.1007/s00595-017-1559-x

14 Pan Y, Wang WD and Yago T: Transcriptional regulation of podoplanin expression by PROX1 in lymphatic endothelial cells. Microvasc Res 94: 96-102, 2014. PMID: 24944097. DOI: 10.1016/j.mvr.2014.05.006

15 Lecompte S, Pasquetti G, Hermant X, Grenier-Boley B, Gonzalez-Gross M, De Henauw S, Molnar D, Stehle P, Beghin L, Moreno LA, Amouyel P, Dallongeville J and Meirhaeghe A: Genetic and molecular insights into the role of PROX1 in glucose metabolism. Diabetes 62(5): 1738-1745, 2013. PMID: 23274905. DOI: $10.2337 / \mathrm{db} 12-0864$

Received March 21, 2019

Revised May 15, 2019

Accepted May 29, 2019 